\title{
FIB Damage in Silicon: Amorphization or Redeposition?
}

\author{
S. Rajsiri, B. W. Kempshall, S.M. Schwarz, and L. A. Giannuzzi
}

Department of Mechanical, Materials, and Aerospace Engineering, University of Central Florida, 4000 Central Florida Blvd., Orlando, FL, 32816-2450

The Focused Ion Beam (FIB) instrument has been utilized for site-specific specimen preparation for a wide range of analytical techniques due to its ability to achieve high spatial resolution imaging, milling, and deposition [1,2]. The understanding of FIB damage is important to ensure that the region being analyzed is indeed representative of the material, and is not due to a specimen preparation artifact. The interaction between the incident ions (e.g., $\mathrm{Ga}^{+}$) and the target material during FIB operation (e.g., imaging or milling mode) may lead to surface damage and consequently limit the ability to achieve high quality high-resolution TEM images. Amorphization of a FIB milled crystalline surface may occur due to sufficient atom displacement within the collision cascade resulting in the loss of long-range order when the density of point defects reaches a critical value [3]. Redeposition of sputtered atoms has also been reported as a result of FIB milling [4]. The propensity for redeposition increases when FIB milling is performed in a confined and/or a high aspect ratio trench, or when FIB conditions are used that contribute to factors that increase the sputtering rate (e.g., using a higher beam current) [5]. Observations have shown that FIB milling with $\mathrm{Ga}$ at an energy of $30 \mathrm{keV}$ will produce amorphization damage along a Si side-wall that is $\sim 28$ $\mathrm{nm}$ thick and up to $20 \mathrm{wt} \%$ Ga may be present within the damage region [6]. Previous work in our lab has shown that the side-wall damage thickness in Si varies with beam current [7]. In addition, while significant amounts of $\mathrm{Ga}$ were observed in the side-wall damage [8], Ga was not detectable in side-wall damage when Si was FIB milled using gas assisted etching (GAE) [9]. The following study was performed in an attempt to better understand FIB damage. In this study, three square trenches $\left(2 \times 2 \mu \mathrm{m}^{2}, 4 \times 4 \mu \mathrm{m}^{2}\right.$, and $\left.6 \times 6 \mu \mathrm{m}^{2}\right)$ were FIB milled to $1 \mu \mathrm{m}$ in depth in a (100) Si wafer using an FEI 200TEM FIB workstation equipped with a Ga liquid metal ion source and an Omniprobe in-situ $\mathrm{W}$ probe. An accelerating voltage of $30 \mathrm{keV}$ and a beam current of $1000 \mathrm{pA}$ was used to mill the trenches. The specimen was removed from the FIB and sputter-coated with Cr to preserve the FIB milled damage layers. The specimen was put back into the FIB and the trenches were filled with CVD Pt deposition using a beam current of $100 \mathrm{pA}$. A cross-section TEM specimen was prepared across the trenches using the in-situ FIB lift-out method [10]. The specimen was observed using a Philips EM430 operating at $300 \mathrm{keV}$. A bright field (BF) TEM image of the trenches is shown as FIG. 1 (a). A BF image from the top of the wafer is shown in FIG. 1(b). A BF image from the side-wall of the middle trench is shown in FIG. 1(c). Note that the side-wall damage clearly consists of two regions with different contrast. This layer clearly indicates that the side-wall damage consists of two regions: (i) an amorphization layer and (ii) a redeposition layer. X-ray energy dispersive spectrometry (XEDS) results showed that significant amounts of Ga (up to $\sim 30 \mathrm{wt} \%$ ) was observed in the redeposition layer, while the presence of $\mathrm{Ga}$ is barely detectable within the limits of the XEDS technique in the amorphization region. The darker region in the redeposition region is consistent with atomic number contrast due to the presence of $\mathrm{Ga}$ and $\mathrm{Si}$ in the redeposition region. The redeposition region increases toward the mouth of the trench. This is consistent with the cosine distribution of sputtered material from within the trench. The thickness of the amorphization layer on the top of the wafer $(\sim 70 \mathrm{~nm})$ is thicker than just the amorphization damage on the side-wall $(\sim 14 \mathrm{~nm})$ which is consistent with ion beam/interactions theory. 
Therefore, the amorphization layer may be considered as an intrinsic behavior of the ions interacting with the target. However, any significant amount of Ga (e.g., > $1 \mathrm{wt} \%$ ) that is observed in the FIB damage region is a redeposition artifact. This is consistent with previous observations whereby Ga was not observed in FIB trenches prepared in conjunction with GAE, since GAE aids in removing redeposition artifacts [9]. In addition, it is noted that no Ga was observed in the TEM specimen using the in-situ lift-out method since any sputtered material may readily be removed by the FIB vacuum system. Since ex-situ lift-out specimens are prepared to electron transparency within a trench, they are more likely to suffer from redeposition artifacts. Thus, we conclude that any Ga observed in TEM specimens may be do to redeposition artifacts [11].

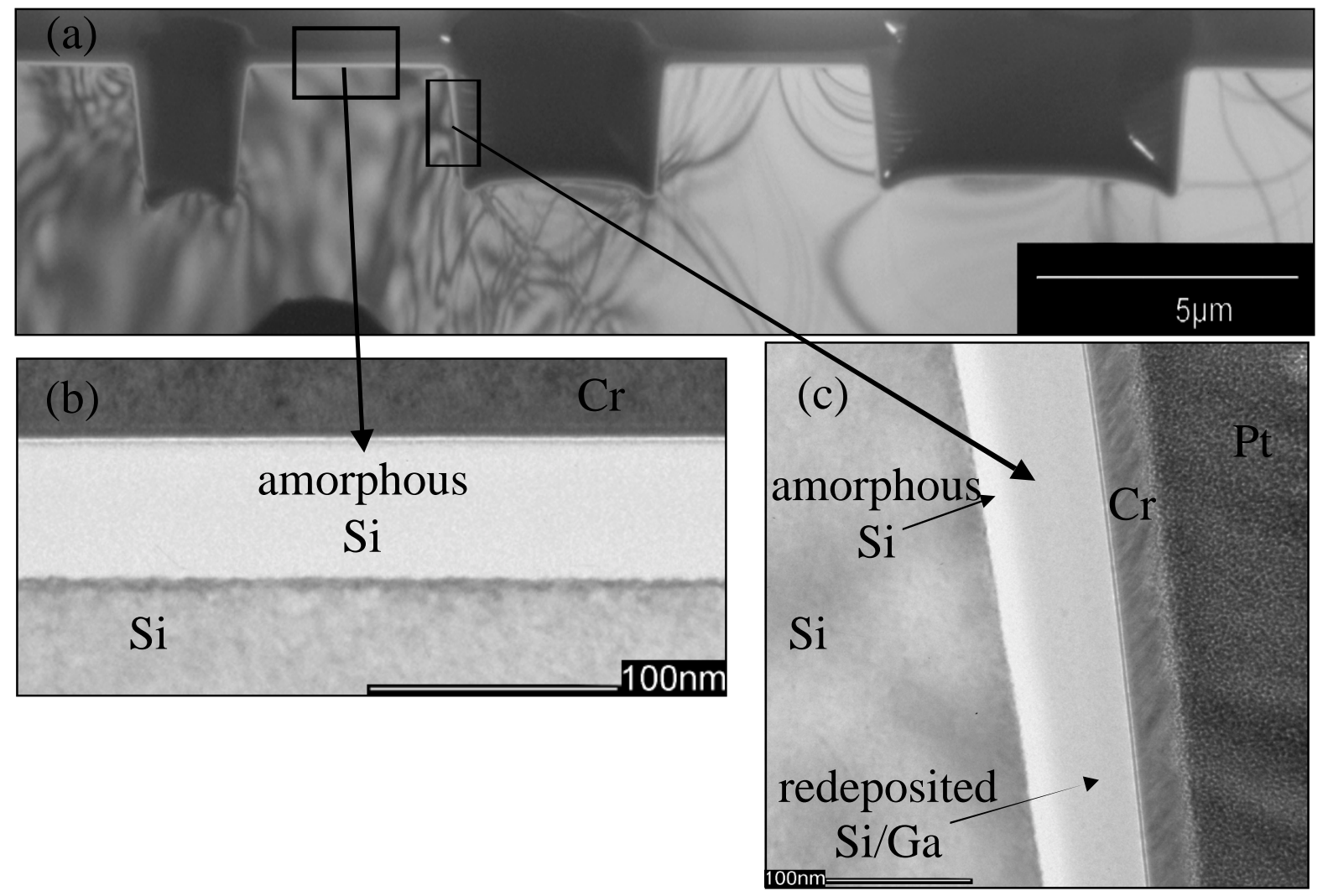

FIG. 1. (a) TEM image of trenches FIB milled into Si. (b) TEM image of the top surface of Si. (c) TEM image of a trench side-wall.

\section{References}

[1] L.A. Giannuzzi, et al., Mat. Res. Soc. Symp. Proc., 480 (1997) 19.

[2] F.A. Stevie et al., Surf. Interface Anal. 31 (2001) 345.

[3] H. Cerva et al., Inst. Phys. Conf. Ser 134 (1993) 133.

[4] Abramo et al., Int. Symp. For Testing and Failure Analysis 20 (1994) 439.

[5] B.I. Prenitzer et al., Microsc. Microanal. 6 (Suppl. 2 ) (2000) 502.

[6] D.W. Susnitzky et al, Microsc. Microanal. 4 (Suppl. 2) (1998) 656.

[7] C.Urbanik Shannon et al., Microsc. Microanal. 5 (Suppl. 2) (1999) 740.

[8] C. Urbanik Shannon, et al., Proceedings of the Second Conference of the IUMAS (2000) 177.

[9] C. Uranik Shanno, M.S. Thesis, University of Central Florida

[10] T. Kamino et al., Microsc. Microanal. 6 (Suppl. 2 ) (2000) 510.

[11]The support of NSF DMR \#9703281 and Omniprobe is greatly appreciated. 